\title{
Baseline Moisture Resistance of PWP Cement Composite Boards Reinforced with Internal Glass Fiber Reinforcement under Accelerated Wet-Dry Aging
}

\author{
Woraphot Prachasaree, ${ }^{1}$ Sitthichai Piriyakootorn, ${ }^{1}$ \\ Suchart Limkatanyu, ${ }^{1}$ and Abideng Hawa ${ }^{2}$ \\ ${ }^{1}$ Department of Civil Engineering, Faculty of Engineering, Prince of Songkla University, Hat Yai, Songkhla 90112, Thailand \\ ${ }^{2}$ Department of Civil Engineering, Princess of Naradhiwas University, Amphur Muang, Narathiwat 96000, Thailand
}

Correspondence should be addressed to Woraphot Prachasaree; pworaphot@eng.psu.ac.th

Received 6 June 2014; Revised 22 July 2014; Accepted 22 July 2014; Published 5 August 2014

Academic Editor: Hui Shen Shen

Copyright (c) 2014 Woraphot Prachasaree et al. This is an open access article distributed under the Creative Commons Attribution License, which permits unrestricted use, distribution, and reproduction in any medium, provided the original work is properly cited.

\begin{abstract}
The purpose of this study was to improve the mechanical characteristics and moisture resistance of parawood particle (PWP) cement composite board developed by the authors. PWP cement composites were prepared with a mixture containing $15.0 \%$ parawood particles, $43.0 \%$ cement, $41.2 \%$ water, and $0.86 \% \mathrm{CaCl}_{2}$ (by weight) and the PWP cement composite boards were internally reinforced with bidirectional glass fiber woven roving $\left[0^{\circ} / 90^{\circ}\right]$. The board properties were evaluated under accelerated aging with wet-dry cycles to establish the durability and moisture resistance and the effect on flexural strength of the composite boards. The mechanical characteristics determined were the equivalent modulus of rupture (eMOR), the equivalent modulus of elasticity (eMOE), and the deformability factor (DF). The experimental results suggest that the strength and stiffness of the PWP composite boards with internal reinforcement are four times higher than those of the original PWP composite boards under accelerated aging based on 100 wet-dry cycles, implying better durability of the boards in outdoor use. The results provide a baseline to which improved formulations and reinforcements or designs can be compared using the same measurement methodology.
\end{abstract}

\section{Introduction}

Para rubberwood is extensively cultivated in South-East Asian countries, where most of the world's rubber tree plantations are currently located. The trees have a limited service life due to their eventual loss of productivity, and they need to be felled to make land available for replanting. Only a small part of the wood materials from felled trees can be used as timber, and converting trees to timber generates a large though biodegradable waste stream of sawdust, chips, trimmings, branches, and even whole trunks. These can be used as fuel or disposed of in landfills, but increasing the range of valueadded uses as filler or fibrous reinforcement in composites would be a better option, for both economic and environmental reasons. Recently, there has been considerable interest and active research into a type of material used for construction and decorative materials known as cement composites.
In previous research on cement composites incorporating wood wastes, concrete mixes with ground hazelnut shell, wood sawdust, and tea wastes have been experimentally investigated and their mechanical properties determined [1]. When wood particles from construction wastes were bonded with cement to form composites, the load-deflection characteristics demonstrated elastoplastic behavior [2]. Composite boards with rice straw and wood particles have also been developed for commercial use as insulation boards [3] and the properties of composites incorporating wood particles from CCA-treated southern yellow pine in cement have been evaluated in compressive tests [4]. Further, the use of thermomechanical pulp (TMP) has been compared with both unbleached and bleached kraft pulp as a fiber reinforcement in cement composites [5, 6]. The flexural behavior and performance of cement composites reinforced with weld square geogrid mesh were also evaluated in [7] and 
it was found that the first crack and ultimate load capacity increased with an increase in the amount of reinforcement. Additionally, the effect of fiber alignment on the mechanical behavior of cement based matrix composites reinforced with aligned pulp fiber sheets was investigated in [8].

The durability of a lightweight concrete composite, based on wood chippings, was investigated under various environmental exposures in [9] and the use of wood sawdust and limestone dust as fine aggregates leads to the development of a lightweight composite product in [10]. AR glass and PE fabric reinforced composites have also been developed and studied under a three-point bending condition using both a static and drop weight impact system. There were three main test parameters including fabric type, specimen orientation, and drop height of the hammer. The impact load capacity and deflection were found in [11] to be highly influenced by the direction of the fabric in the composites. In [12], cement composites reinforced with Sisal Organosolv pulp and polypropylene fibers were evaluated using accelerated carbonation aging and soak-dry cycles. The influence of reinforcement with metal and woven synthetic nets on the properties of medium density fiberboard was studied in [13] and it was found that the mechanical properties of medium density fiberboard were improved by such reinforcement. Cement composites with parawood particle wastes were found to be acceptable for use in structural members with a low load carrying capacity and for some other construction uses in [14].

However, the use of Para rubberwood product wastes in building materials is still not commercially significant, partly because there are gaps in knowledge that research can address. In particular, the moisture resistance of such products is of concern in construction applications and needs further assessment and potential improvement from the current baseline. This study documents that baseline and its main objectives were to develop parawood particle cement composite boards with internal glass fiber reinforcement and to study their performance and durability under wet-dry cycles. This study will aid the development of building and construction uses for parawood cement composites and will establish the baseline from which further improvements in moisture resistance capable of expanding the potential uses of such composites can be measured.

\section{Materials}

In this study, waste PWP in their original form were mixed with ordinary Portland cement to make PWP cement composites. The PWP were obtained by collecting wood dust, created in the furniture industry during the surface abrasion of parawood boards. An image of the PWP is shown in Figure 1. The PWP sizes ranged up to $1180 \mu \mathrm{m}$. Their specific gravity and moisture content were determined according to ASTM D 1037-99 [15]. The chemical compositions of the PWP and Portland cement were determined using X-ray fluorescence spectrometry. The ordinary Portland cement (OPC-ASTM Type I cement) in this study was obtained from a local supplier. The physical properties and chemical

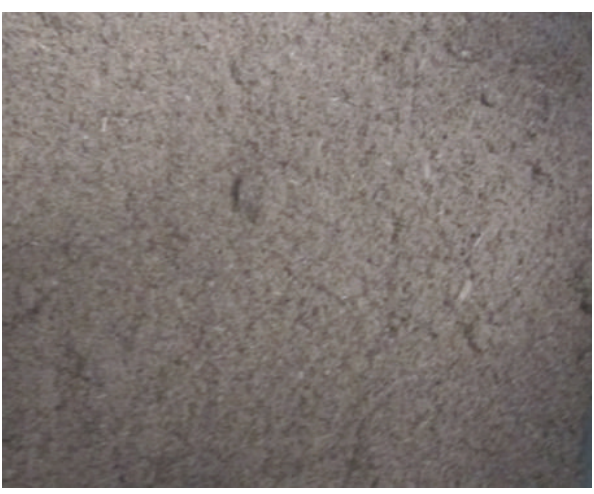

Figure 1: Parawood particle particle.

TABLE 1: Physical properties and chemical composition.

(a) Chemical composition (\%)

\begin{tabular}{lccccccc}
\hline Materials & $\mathrm{Al}_{2} \mathrm{O}_{3}$ & $\mathrm{CaO}$ & $\mathrm{SiO}_{2}$ & $\mathrm{Fe}_{2} \mathrm{O}_{3}$ & $\mathrm{MgO}$ & $\mathrm{K}_{2} \mathrm{O}$ & $\mathrm{SO}_{3}$ \\
\hline Portland cement & 2.56 & 70.85 & 11.59 & 4.40 & 1.17 & 1.37 & 4.20 \\
PWP & 0.28 & 16.84 & 1.42 & 0.71 & 0.68 & 2.70 & 0.82 \\
\hline
\end{tabular}

(b) Physical properties

\begin{tabular}{lcccc}
\hline Materials & $\begin{array}{c}\text { Specific } \\
\text { gravity }\end{array}$ & $\begin{array}{c}\text { Moisture } \\
\text { content } \\
\text { (\% weight) }\end{array}$ & $\begin{array}{c}\text { (\% weight) } \\
\text { Loss on } \\
\text { ignition }\end{array}$ & $\mathrm{pH}$ \\
\hline Portland cement & 3.15 & 0.34 & 1.05 & 12 \\
PWP & 1.56 & 2.92 & 74.7 & 8 \\
\hline
\end{tabular}

compositions are given in Table 1 . It should be noted that the ratio of combined $\mathrm{CaO}$ and $\mathrm{K}_{2} \mathrm{O}$ to $\mathrm{SiO}_{2}$ is higher than suggested by European Standard EN 197-1:2000 [15]. Thus, high alkalinity and hydration activity can be expected in these parawood cement composites. The glass woven roving used in this study consisted of fibers held together in a bidirectional arrangement. The glass strands were orientated in both the horizontal and the perpendicular $\left[0^{\circ} / 90^{\circ}\right]$ directions with a density of $800 \mathrm{~g} / \mathrm{m}^{2}$. The tensile strength of individual glass fibers ranged from 1300 to $3400 \mathrm{MPa}$. The tensile modulus of elasticity was around $73 \mathrm{GPa}$.

\section{PWP Cement Composite Boards}

The mix proportions of the composite used to produce the boards were $15.0 \%$ parawood particles, $43.0 \%$ cement, $41.2 \%$ water, and $0.86 \% \mathrm{CaCl}_{2}$ (by weight) and represent a reasonable compromise between high strength and high content of wood particles [16]. Composite boards with dimensions of $300 \times 300 \times 20 \mathrm{~mm}$ were produced from PWP and cement from the above formulation. The boards had an apparent specific gravity of 1.324 (ignoring porosity) based on weight and dimensions. To produce the boards, first, the PWP were mixed with Portland cement; then the water and $\mathrm{CaCl}_{2}$ were added. The slurry was manually stirred thoroughly until it was uniform. The mixture was then poured into steel molds and the glass fabric reinforcement was placed in the mixture. 


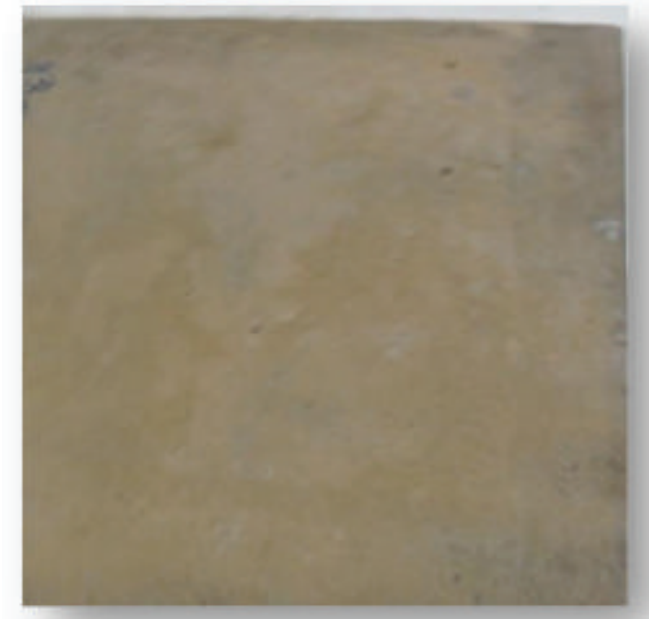

Top view: board samples

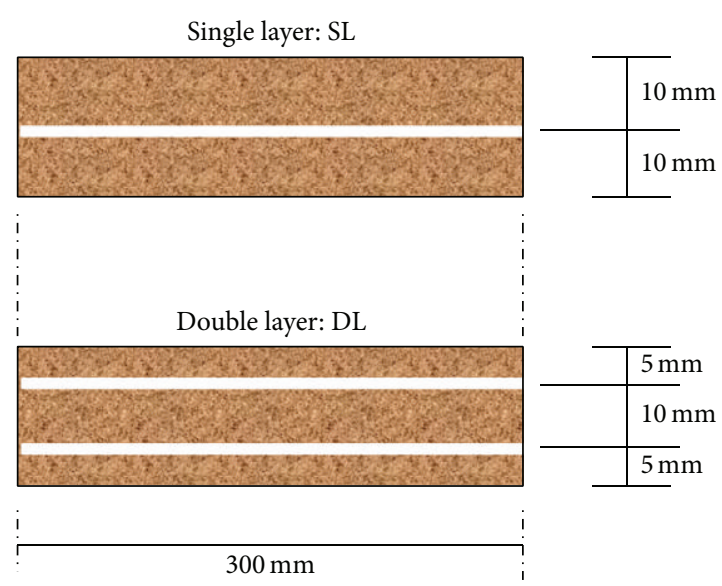

Cross section

FIGURE 2: PWP cement composite boards reinforced with glass fabric.

The composite boards were cold-pressed to attain the desired dimensions. Two different patterns of internal reinforcement were used as shown in Figure 2.

After 24 hours at room temperature, the PWP cement composite boards were demolded and cured under damp burlap for 3 days before being tested and wet-dry aged. To evaluate the strength of control samples, a flexural test was performed on the composite boards without accelerated aging, after they had been cast and cured for 4 days. In total, twenty-four test samples of the PWP cement composite boards (6 samples without reinforcement, 9 samples with single layer reinforcement, and 9 samples with double layer reinforcement) were prepared for performance and durability tests. For PWP cement composite boards with single layer reinforcement, it should be noted that the glass reinforcement was positioned in the middle of the PWP board thickness. In general, the tensile properties of cement composite materials are weak. Glass fiber reinforcement should be placed on the tension side of the cement composite boards. However, it can make users confused that the PWP cement boards must be properly installed in the correct side of the PWP cement board surfaces. Thus, the glass reinforcement was positioned in the middle of thickness for this study.

\section{Accelerated Wet-Dry Aging}

This study was conducted in order to establish the durability and moisture resistance of the cement composite boards. The wet-dry accelerated aging employed was based on recommendations contained in the EN 494 Standards [17]. Each cycle involved the sample being immersed in water for 5 hours 50 minutes at $20 \pm 5^{\circ} \mathrm{C}$, followed by air drying for 10 minutes, then 5 hours 50 minutes in a ventilated oven at $70 \pm 5^{\circ} \mathrm{C}$, followed by air drying for 10 minutes. These cycles were repeated 50 or 100 times before flexural testing. The system of accelerated aging is illustrated in Figure 3.

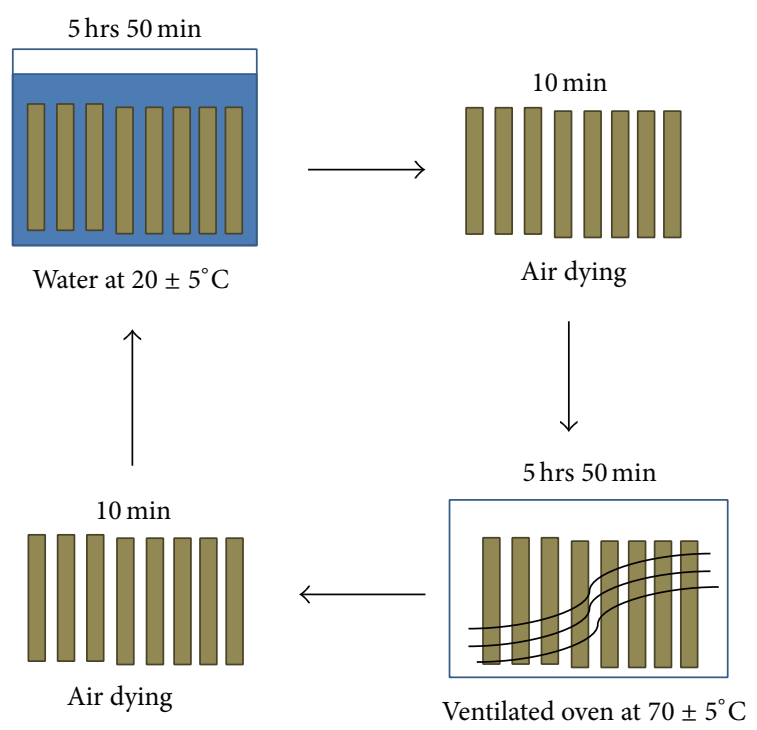

Figure 3: Accelerated wet-dry cycling process.

\section{Experiment and Evaluations}

The flexural mechanical properties of the PWP cement composite boards were investigated, as indicators of structural performance. The board samples were tested in a three-point bending scheme with a support test span of $240 \mathrm{~mm}$. A concentrated line load was applied from above, at the midpoint of the test span of the samples. A linear variable differential transducer (LVDT) was mounted underneath at the midpoint to determine the induced deflection. A data acquisition system recorded the loads and deflections at a high sampling rate during each test. The setup is schematically illustrated in Figure 4. To determine the flexural strength of the PWP cement composite board samples, the equivalent modulus of 
TABLE 2: Physical and mechanical properties of PWP cement composite boards.

\begin{tabular}{|c|c|c|c|c|c|c|c|}
\hline \multirow{2}{*}{$\begin{array}{l}\text { Reinforcement } \\
\text { layer }\end{array}$} & \multirow{2}{*}{ Wet-dry cycles } & \multirow{2}{*}{ Number of samples } & \multirow{2}{*}{ Density $\left(\mathrm{kg} / \mathrm{m}^{3}\right)$} & \multicolumn{4}{|c|}{ Average mechanical properties } \\
\hline & & & & Average max load $(\mathrm{kN})$ & eMOR (MPa) & eMOE (MPa) & DF factor \\
\hline \multirow{2}{*}{-} & 50 & 3 & 1288 & 0.609 & 7.31 & 1802 & 1.474 \\
\hline & 100 & 3 & 1158 & 0.400 & 4.80 & 1050 & 1.451 \\
\hline \multirow{3}{*}{ Single } & 0 & 3 & 1340 & 1.152 & 13.82 & 3657 & 2.230 \\
\hline & 50 & 3 & 1332 & 0.982 & 11.78 & 3610 & 2.205 \\
\hline & 100 & 3 & 1323 & 0.691 & 8.29 & 2215 & 1.522 \\
\hline \multirow{3}{*}{ Double } & 0 & 3 & 1357 & 2.170 & 26.00 & 6290 & 2.360 \\
\hline & 50 & 3 & 1354 & 1.710 & 20.50 & 5836 & 2.290 \\
\hline & 100 & 3 & 1349 & 1.627 & 19.52 & 5215 & 1.972 \\
\hline
\end{tabular}

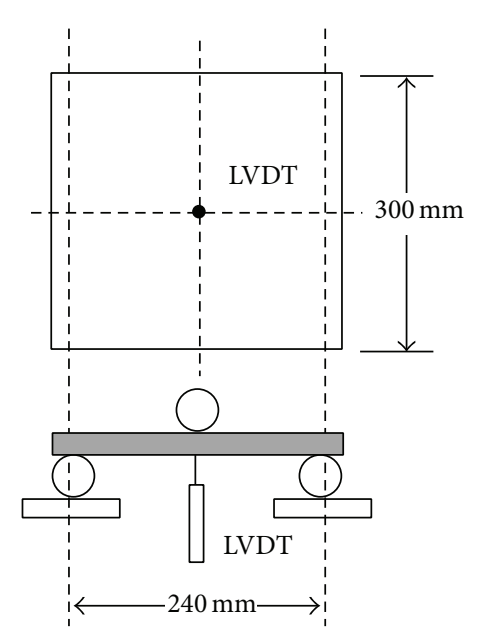

FIGURE 4: Schematic representation of the three-point bending test.

rupture (eMOR) was used. In general, the flexural strength of cement composite boards is evaluated from the maximum applied load before board rupture. The eMOR for the PWP cement composite boards is given in (A.1), Appendix A. In addition, the equivalent modulus of elasticity (eMOE) based on the slope $(P / \Delta)$ of the experimental load and deflection relation in the linearly elastic range is determined using (A.2), Appendix A.

The ductility or deformability of PWP cement composite boards can be evaluated by using experimental data in the post elastic range. Generally, the post elastic behavior of PWP cement composite boards is indicated by the deformability factor (DF). The DF of PWP cement composite boards can be defined as the ratio of deflection (or curvature or rotation, depending on which of these is observed) at the ultimate point $\left(\delta_{u}\right)$ to those values at the elastic point $\left(\delta_{y}\right)$ as given in

$$
\mathrm{DF}=\frac{\delta_{u}}{\delta_{y}},
$$

where $\delta_{y}$ and $\delta_{u}$ are elastic and ultimate deflections at the mid composite board. In general, the relations between load and deflection are not composed of linear segments. The elastic and ultimate deflections in (1) are not straightforward. To determine the DF, the procedure set out in EN 1995-1-1 [18]
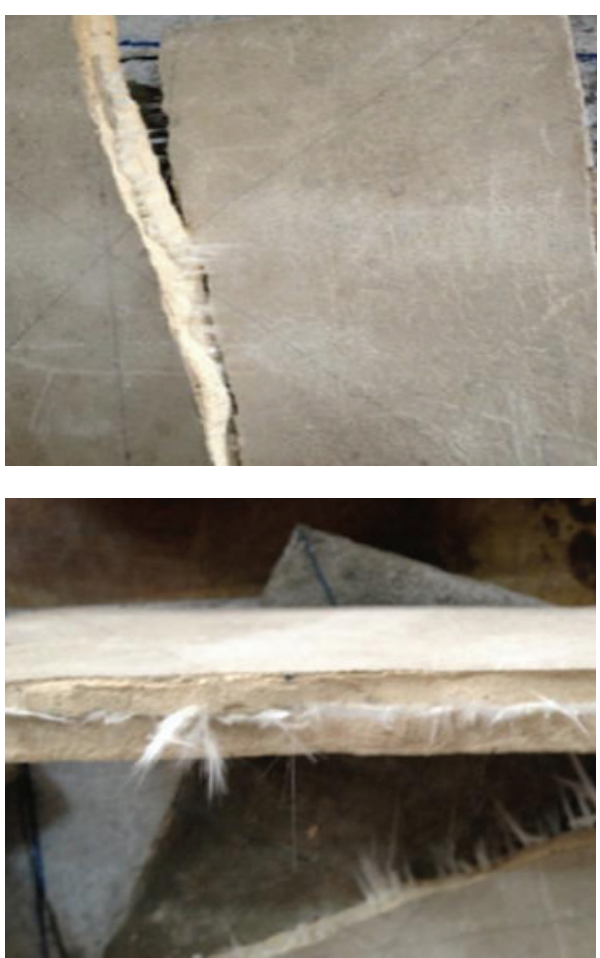

FIgURE 5: Failure mode of the PWP cement composite boards.

was modified and used to define elastic and ultimate points in this study (Appendix B).

\section{Results and Discussion}

Based on experimental results, the density of PWP cement composite boards with glass reinforcement is higher than that of PWP cement composite boards without reinforcement. Under accelerated wet-dry aging, the density of PWP cement composite boards decreases as the number of aging cycles increases as shown in Table 2. It should be that the accelerated wet-dry aging has led to significant reduction in density and strength particularly in PWP cement composite boards without internal reinforcement. The results are summarized along with the relevant physical properties in Table 2. 

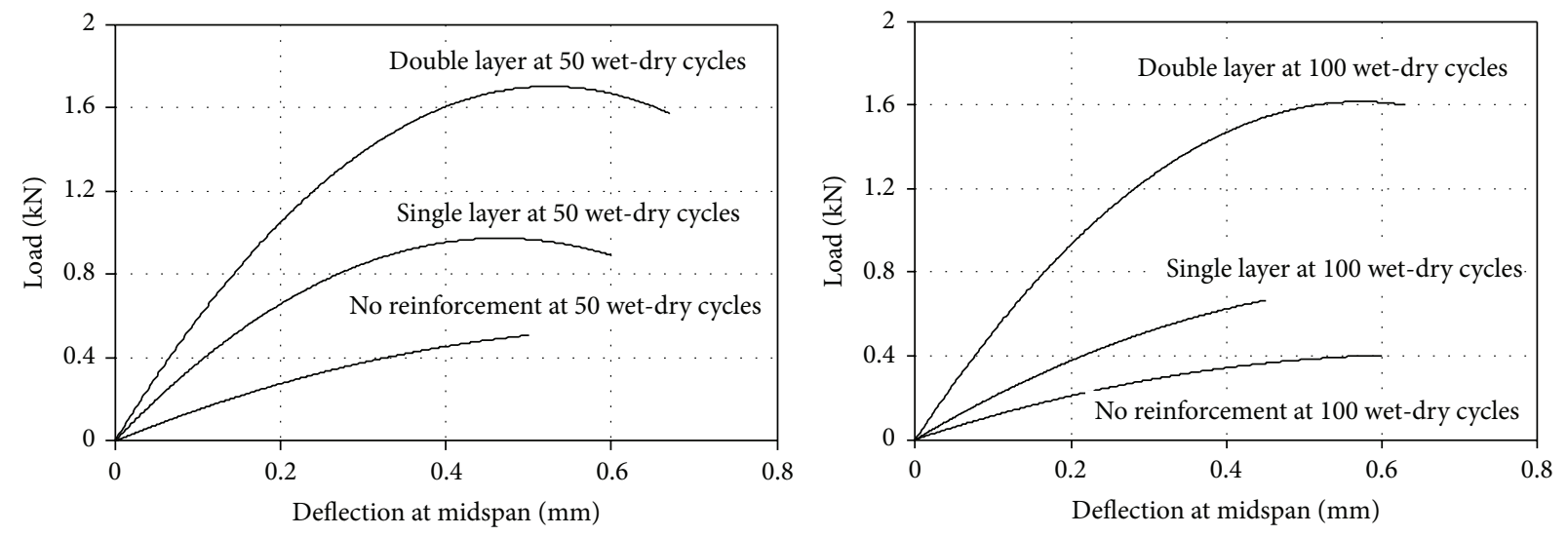

FIGURE 6: Load-deflection relations: effect of reinforcement.

The mechanical properties determined from the flexural experiments were the equivalent modulus of rupture (MOR), equivalent modulus of elasticity (eMOE), and deformability factor (DF).

6.1. Behavior and Failure Mode. As expected, the mechanical properties degraded along with the decrease in the amount of glass reinforcement and the increase in the number of wetdry cycles. From the experimental results, it can be clearly observed that the mechanical properties of the PWP cement composite boards under wet-dry cycles can be improved by using internal glass reinforcement. For the PWP cement composite boards without any internal reinforcement both prior to and after wet-dry cycling, the deflection increased almost linearly with the applied load, until the first major crack emerged. During testing, the formation of the first major crack was typically observed, immediately preceding the sudden failure of the board, which was similar to the failure behavior of the PWP cement composite boards with internal reinforcement. However, smaller cracks were also found in the samples with internal reinforcement.

The failure mode of the PWP cement composite boards with internal reinforcement is summarized in Figure 5. In addition, the load-deflection responses of the PWP cement composite boards under wet-dry aging are presented in Figures 6 and 7.

6.2. Effect of Wet-Dry Cycles and Reinforcement on Strength and Stiffness. The flexural strengths of the PWP cement composite boards with single and double layers of glass fabric reinforcement were compared with those of the boards without reinforcement under wet-dry aging conditions and were found to be, respectively, 1.6-1.7 and 3.0-4.0 times those of the composite boards without reinforcement, as shown in Figure 6. The average percent increase in the strength of the PWP cement composite boards with internal reinforcement was summarized as shown in Figure 7. In addition, the flexural stiffness was observed to be proportional to the slope of the load-deflection curve and increased directly with the increase in internal glass reinforcement. However, the flexural strength and stiffness indicated a trend of decrease

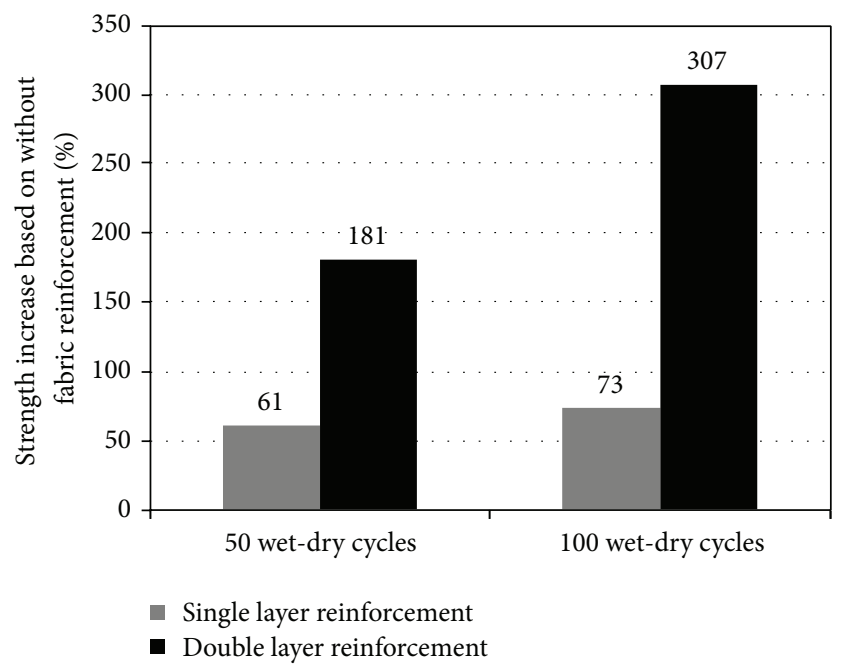

FIgURE 7: Percent increase in strength of PWP cement composite boards.

with increasing aging cycles as shown in Figure 8. Thus, aging by wet-dry cycling significantly reduced the strength, stiffness, and postcracking. Based on previous research [16], the strength reduction of PWP cement composite board without internal reinforcement under wet-dry aging has been found to be close to $70 \%$ after 100 aging cycles. In this study, however, the strength reduction at 100 wet-dry cycles was found to be $40 \%$ and $25 \%$, respectively, for the PWP cement composite boards with single and double layers of reinforcement. Summary of the strength reduction under accelerated wet-dry cycles was presented in Figure 9. The experimental results show that internal glass fabric reinforcement significantly improves the overall mechanical properties of the PWP cement composite boards.

6.3. Effect of Wet-Dry Cycles and Reinforcement on the Equivalent Modulus of Elasticity. As can be seen from Table 2, the eMOE was less sensitive to aging by wet-dry cycling than the average maximum load capacity or the eMOR. The eMOE shows a less significant change at 50 wet-dry cycles, and there 

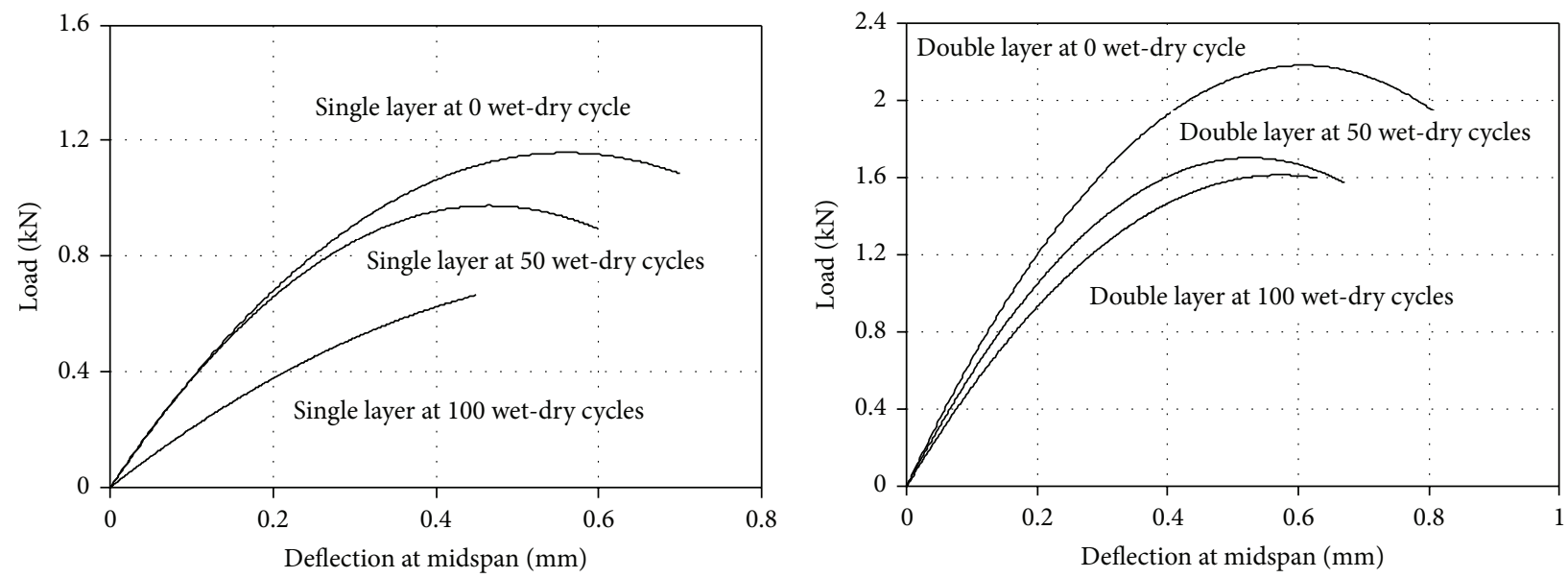

FIGURE 8: Load-deflection relations: effect of wet-dry cycles.

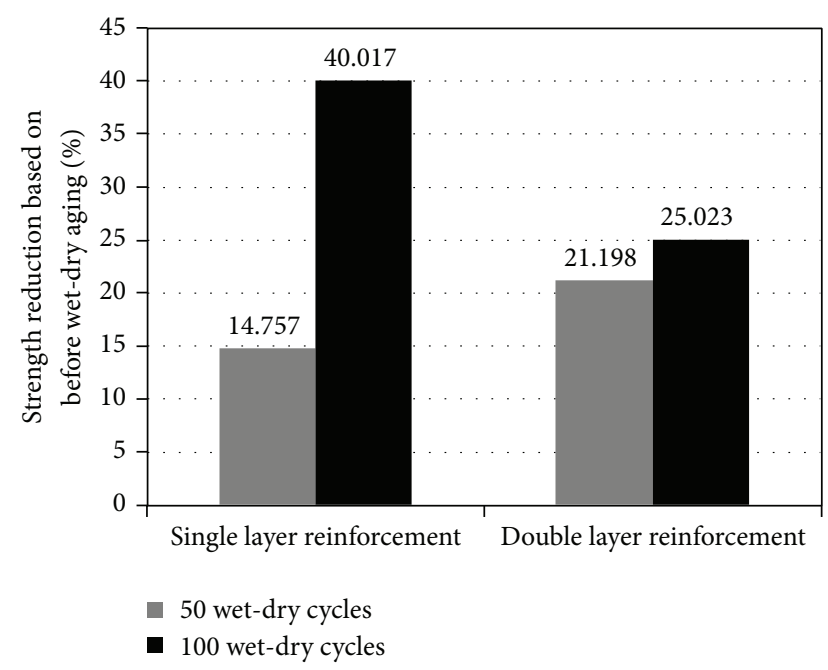

FIGURE 9: Strength reduction of PWP cement composite boards under wet-dry aging.

was only a small decrease in the eMOE found between 50 and 100 wet-dry cycles. It should be noted that the trend of eMOE reduction under wet-dry aging is quite different for the PWP cement composite boards with and without internal reinforcement, since the load-deflection behavior of the PWP cement composite boards without internal reinforcement was almost linear until brittle failure, particularly after 100 wet-dry cycles [16]. The internal glass fabric reinforcement therefore serves to reduce the brittle behavior and increase the ductility of the PWP cement composite board.

6.4. Deformability Factor (DF). The trend of the DF was similar to that of the eMOE for the PWP cement composite boards with internal glass fiber reinforcement. The maximum reductions in the DF obtained from the PWP cement composite boards after 100 wet-dry cycles were $31.7 \%$ and $16.4 \%$, respectively, for single and double layer reinforcement. The
DF showed little change at 50 cycles but was significantly reduced after 100 wet-dry cycles.

\section{Conclusions}

Composite boards prepared from PWP particles and cement (35\% PWP content relative to cement), with and without internal glass fabric reinforcement, were tested for the effects of accelerated aging by wet-dry cycles, on their mechanical flexural properties. All of the mechanical properties of the PWP cement composite boards with double layer reinforcement increased significantly, indicating that these boards had improved moisture resistance and are suitable for outdoor applications. After 100 wet-dry cycles, the flexural strength and stiffness of boards with double layer reinforcement were about four times higher than those of boards without internal reinforcement. These composite boards may therefore be suitable for indoor use other than as structural members.

\section{Appendices}

\section{A. Equivalent Modulus of Rupture and Elasticity}

The equivalent modulus of rupture (eMOR) can be determined as follows:

$$
\mathrm{eMOR}=\frac{3 P L}{2 b h^{2}}
$$

The equivalent modulus of elasticity (eMOE) can be determined as follows:

$$
\mathrm{eMOE}=\left(\frac{P}{\Delta}\right)\left(\frac{1-v^{2}}{4 b}\right)\left(\frac{L}{h}\right)^{3}
$$

where $P$ is the maximum applied load, $L$ is test span, $h$ is thickness of tested samples, $b$ is the sample width normal to the span, and $v$ is Poisson's ratio $(\approx 0.4)$. 


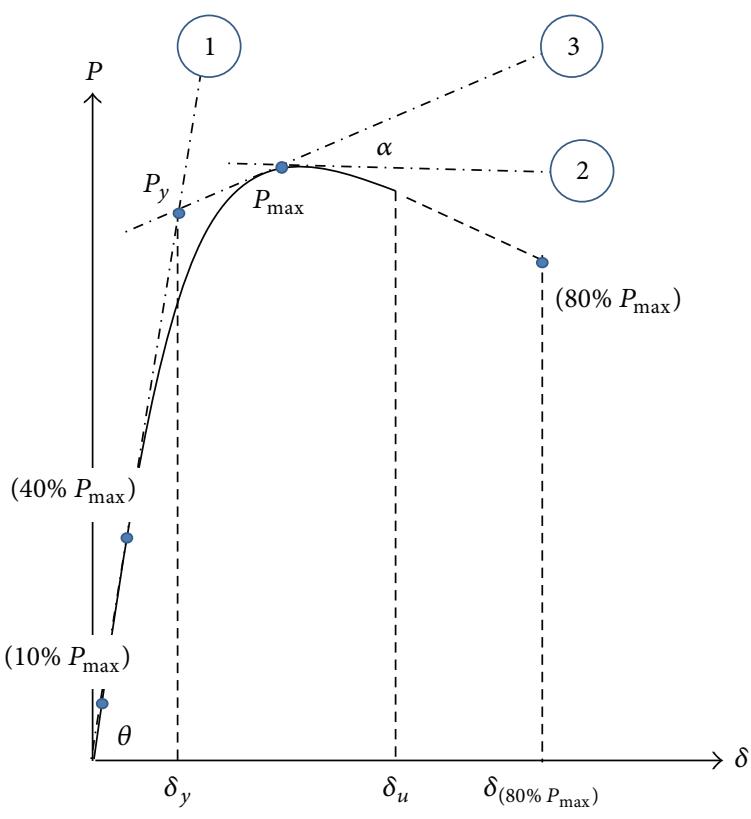

FIgURE 10: Procedure for the ductility factor [17].

\section{B. Deformability Factor}

As shown in Figure 10, the elastic range (fit by line 1) runs from $10 \% P_{\max }$ to $40 \% P_{\max }$ on the load-deflection curve. The peak line (line 2) is a horizontal line passing through the $P_{\max }$ position of the load-deflection relation. To determine an equivalent yield point, line 3 is drawn with inclination angle $\alpha$ at the maximum point of the curve. The equivalent yield point $\left(P_{y}\right)$ is then defined by the intersection of lines 1 and 2 . One has $\operatorname{Tan} \alpha=(\operatorname{Tan} \theta) / 6$.

\section{Conflict of Interests}

The authors declare that there is no conflict of interests regarding the publication of this paper.

\section{Acknowledgments}

The authors would like to acknowledge Associate Professor Seppo Karrila, Ph.D. and Research and Development Office (RDO), Prince of Songkla University, for technical comments and corrections. This study was partially supported by Structural Engineering and Applied Mechanics (STREAM) Research Group under Grant ENG-51-2-7-11-022-S, Department of Civil Engineering, Faculty of Engineering, Prince of Songkla University, Hat Yai, Songkhla, Thailand.

\section{References}

[1] A. Demirbaş and A. Aslan, "Effects of ground hazelnut shell, wood, and tea waste on the mechanical properties of cement," Cement and Concrete Research, vol. 28, no. 8, pp. 1101-1104, 1998.

[2] R. W. Wolfe and A. Gjinolli, "Durability and strength of cementbonded wood particle composites made from construction waste," Forest Products Journal, vol. 49, no. 2, pp. 24-31, 1999.
[3] H. Yang, D. Kim, and H. Kim, "Rice straw-wood particle composite for sound absorbing wooden construction materials," Bioresource Technology, vol. 86, no. 2, pp. 117-121, 2003.

[4] A. Gong, D. P. Kamdem, and R. Harichandran, "Compression tests on wood-cement particle composites made of CCA-treated wood removed from service," in Proceedings of Environmental Impacts of Preservative-Treated Wood Conference, Orlando, Fla, USA, 2004.

[5] B. J. Mohr, H. Nanko, and K. E. Kurtis, "Durability of kraft pulp fiber-cement composites to wet/dry cycling," Cement and Concrete Composites, vol. 27, no. 4, pp. 435-448, 2005.

[6] B. J. Mohr, H. Nanko, and K. E. Kurtis, "Durability of thermomechanical pulp fiber-cement composites to wet/dry cycling," Cement and Concrete Research, vol. 35, no. 8, pp. 1646-1649, 2005.

[7] M. Z. Hossain, M. Rokonuzzaman, and S. Inoue, "Flexural behavior of cement composites panels reinforced with different types of meshes," in Proceedings of the 30th Conference on Our World in Concrete \& Structures, Singapore, 2005.

[8] B. J. Mohr, H. Nanko, and K. E. Kurtis, "Aligned kraft pulp fiber sheets for reinforcing mortar," Cement and Concrete Composites, vol. 28, no. 2, pp. 161-172, 2006.

[9] P. Coatanlem, R. Jauberthie, and F. Rendell, "Lightweight wood chipping concrete durability," Construction and Building Materials, vol. 20, no. 9, pp. 776-781, 2006.

[10] P. Turgut, "Cement composites with limestone dust and different grades of wood sawdust," Building and Environment, vol. 42, no. 11, pp. 3801-3807, 2007.

[11] M. Gencoglu and B. Mobasher, "Static and impact behavior of fabric reinforced cement composites," in Proceedings of the 5th International RILEM Workshop on High Performance Fiber Reinforced Cement Composites (HPFRCC5), pp. 463-470, Cachan, France, 2007.

[12] A. P. Joaquim, G. H. D. Tonoli, S. F. D. Santos, and H. Savastano Jr., "Sisal organosolv pulp as reinforcement for cement based composites," Materials Research, vol. 12, no. 3, pp. 305-314, 2009.

[13] B. Mohebby, F. Tavassoli, and S. Kazemi-Najafi, "Mechanical properties of medium density fiberboard reinforced with metal and woven synthetic nets," European Journal of Wood and Wood Products, vol. 69, no. 2, pp. 199-206, 2011.

[14] P. Woraphot, H. Abideng, and K. Thaniya, "Feasibility study of cement composites with Para wood particle wastes: strength and durability," Global Nest Journal, vol. 13, no. 2, pp. 183-192, 2011.

[15] European Standard, "Cement. Composition, specifications and conformity criteria for common cements," EN 197-1: 2000.

[16] W. Prachasaree, S. Limkatanyu, and A. Hawa, "Parawood particle cement composite boards under accelerated wet/dry cycling and natural aging," Journal of Sustainable Cement-Based Materials, vol. 2, no. 3-4, pp. 227-234, 2013.

[17] European Standard, "Fibre-cement profiled sheets and fittings-product specification and test methods," BS EN 494, 1994.

[18] Eurocode5, Eurocode 5 Design of timber structures, EN1995-11:2004E. 

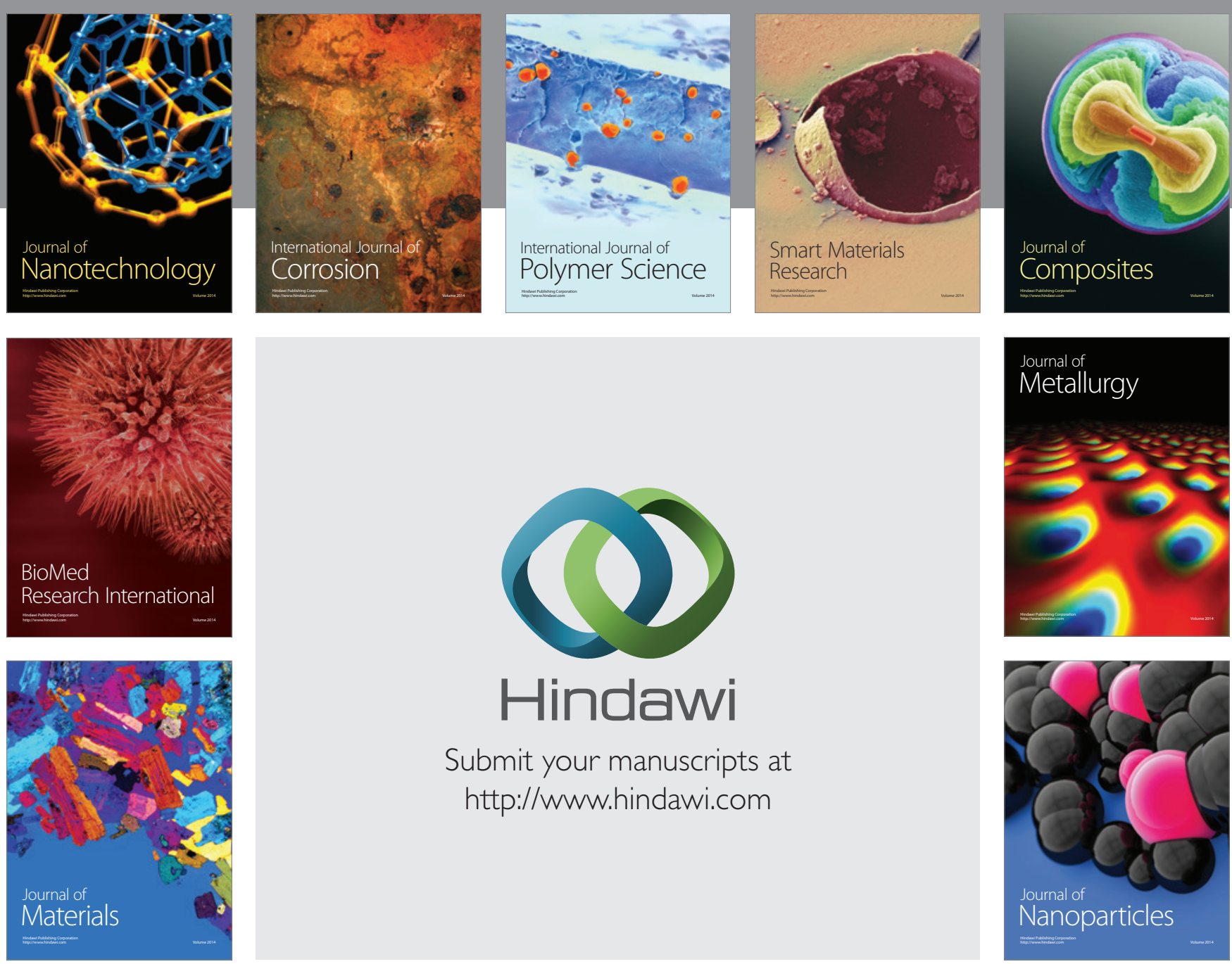

Submit your manuscripts at http://www.hindawi.com
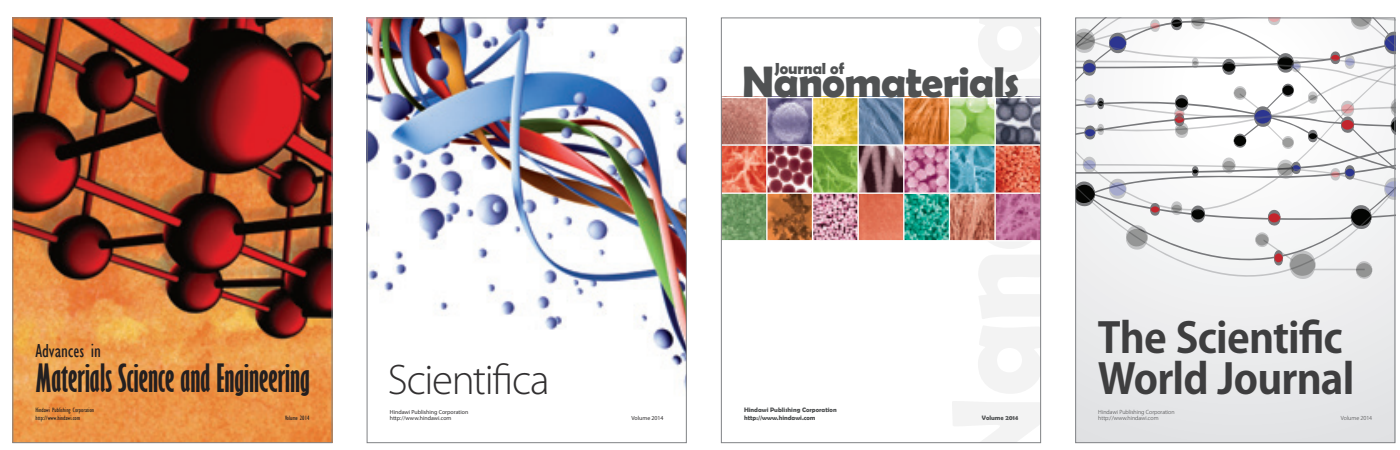

\section{The Scientific World Journal}
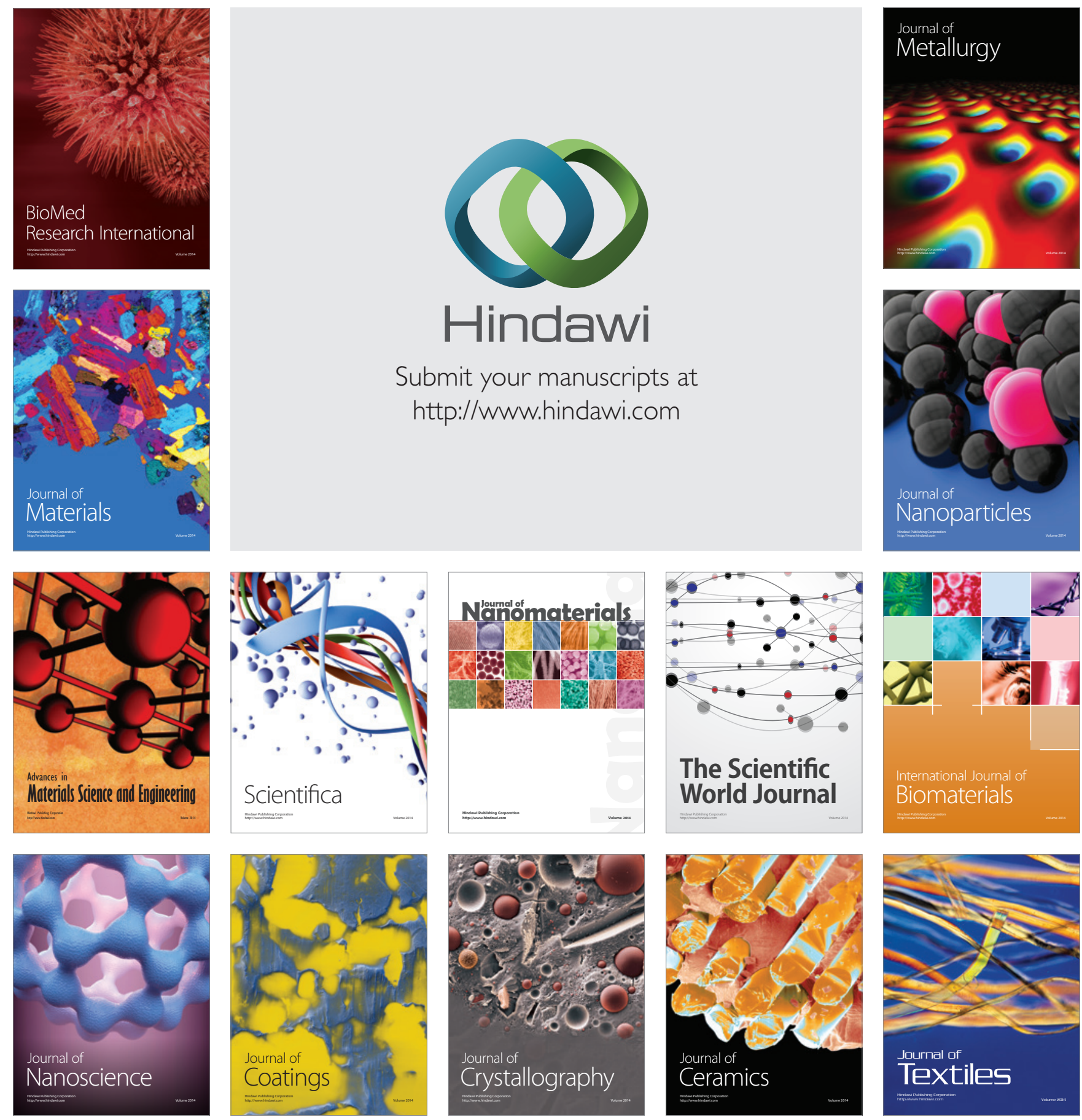